\title{
Electric Potential Structure in the Extraction Region of the Negative Hydrogen Ion Source*)
}

\author{
Shingo MASAKI ${ }^{1)}$, Kenta MAESHIRO ${ }^{2)}$, Katsuyoshi TSUMORI ${ }^{1,3)}$ and Motoi WADA ${ }^{2)}$ \\ 1) Department of Fusion Science, SOKENDAI (The Graduate University for Advanced Studies), Toki, Gifu 509-5292, Japan \\ ${ }^{2)}$ School of Science and Engineering, Doshisha University, Kyotanabe, Kyoto 610-0394, Japan \\ ${ }^{3)}$ National Institute for Fusion Science, Toki, Gifu 509-5292, Japan
}

(Received 9 January 2019 / Accepted 4 June 2019)

\begin{abstract}
Plasma parameters were compared in the extraction region of a negative hydrogen $\left(\mathrm{H}^{-}\right)$ion source between the discharge sustained by tungsten $(\mathrm{W})$ and tantalum $(\mathrm{Ta})$ high temperature cathodes. A hydrogen plasma driven by the Ta filament showed lower electron temperature and higher ratio of positive ion saturation current to negative ion/electron saturation current than a plasma excited by the $\mathrm{W}$ filament. Plasma potential indicated different spatial distribution along the direction of $\mathrm{H}^{-}$ion extraction corresponding to the voltage biased on the plasma electrode. These results are correlated to the intensities of the $\mathrm{H}^{-}$ion extraction current.

(c) 2019 The Japan Society of Plasma Science and Nuclear Fusion Research
\end{abstract}

Keywords: negative hydrogen ion, plasma parameter, Langmuir probe, negative ion extraction, filament material

DOI: $10.1585 /$ pfr. 14.3401136

\section{Introduction}

The amount of negative hydrogen $\left(\mathrm{H}^{-}\right)$ion current which is extracted from a negative ion source driven by DC discharge cathodes is enhanced by seeding cesium (Cs) in the ion source with respect to the ion source of pure $\mathrm{H}_{2}$ discharge, meanwhile suppressing co-extracted electron current [1]. Continuous Cs seeding, however, is necessary to sustain the $\mathrm{H}^{-}$current and seeded $\mathrm{Cs}$ vapor condenses on the walls of the ion source. Some part of the Cs leaks to the accelerator and can induce voltage breakdowns between the accelerator electrodes. For long-period operation, the breakdown will be a serious problem to provide stable beams. For this reason, non-Cs materials have been investigated for efficient $\mathrm{H}^{-}$production [2-4].

Nishiura et al. reported that formation rate of hydrogen vibrationally excited molecules $\left(\mathrm{H}_{2}{ }^{*}\right)$ and $\mathrm{H}^{-}$ion yield increased in hydrogen plasma generated with tantalum (Ta) filaments comparing to tungsten (W) ones [5]. One possible explanation to the effects of Ta filaments on $\mathrm{H}^{-}$ion production is the suppression of associative detachment reaction between $\mathrm{H}^{-}$and hydrogen atoms due to adsorption of hydrogen atoms by Ta deposition on plasma electrodes [6]. Fresh Ta atoms that can absorb atomic hydrogen are supplied to the plasma electrode by evaporation from Ta filament during the hot filament cathode discharge. The absorbed hydrogen atoms can be released as the form of $\mathrm{H}_{2}{ }^{*}$ from the Ta atoms and increase the local $\mathrm{H}_{2}{ }^{*}$ density. Higher density of the $\mathrm{H}_{2}{ }^{*}$ can reduce local electron density through dissociative electron attachment to from $\mathrm{H}^{-}$,

author'se-mail: masaki.shingo@nifs.ac.jp

*) This article is based on the presentation at the 27th International Toki Conference (ITC27) \& the 13th Asia Pacific Plasma Theory Conference (APPTC2018) while lower concentration of atomic hydrogen can further reduce electron density through lower reaction rate of associative electron detachment through collisions with $\mathrm{H}^{-}$. Thus, in the vicinity of the plasma grid (PG) a Ta filament may reduce local electron density. Other plasma parameters, electron temperature and plasma potential, must be altered due to Ta adsorption on PG surface.

A higher ratio of $\mathrm{H}^{-}$ion current $\left(I_{H^{-}}\right)$to co-extracted electron current $\left(I_{\text {ext }}\right)$ was observed by using the Ta filament with respect to $\mathrm{W}$ filament in our previous experiment [7]. The result of the Ta filament operation showed different dependence of the extracted currents, $I_{H^{-}}$and $I_{\text {ext }}$, upon the bias voltage induced to the plasma electrode $\left(V_{b}\right)$, which can affect the $\mathrm{H}^{-}$ion transport in the vicinity of the plasma electrode due to the correlation with the spatial distribution of the plasma potential [8]. For the $V_{b}$ at which the $I_{H^{-}}$takes the maximum intensity, the plasma potential shows a flat spatial distribution against the distance from the plasma electrode. Consequently, the $\mathrm{H}^{-}$ions produced in the driver region upstream of the filter magnetic field [9] can travel to extractor without electrostatic trap [10]. The plasma potential distribution can be more sensitively affect the $\mathrm{H}^{-}$ion transport against the change of $V_{b}$ in $\mathrm{H}^{-}$rich plasmas like the case of cesium seeded ion source comparing with the case of pure $\mathrm{H}_{2}$ discharge [11]. Thus, the investigation of the electric potential structure should give information for better understanding on the behavior of $\mathrm{H}^{-}$ions near the extractor in a negative ion source. The purpose of this study is two-fold; one is to map the local plasma parameters for confirming the effect due to a Ta filament operation as compared to the $\mathrm{W}$ filament operation. Also, the difference of the potential structure in the extraction region of the $\mathrm{H}^{-}$ion source was investigated be- 


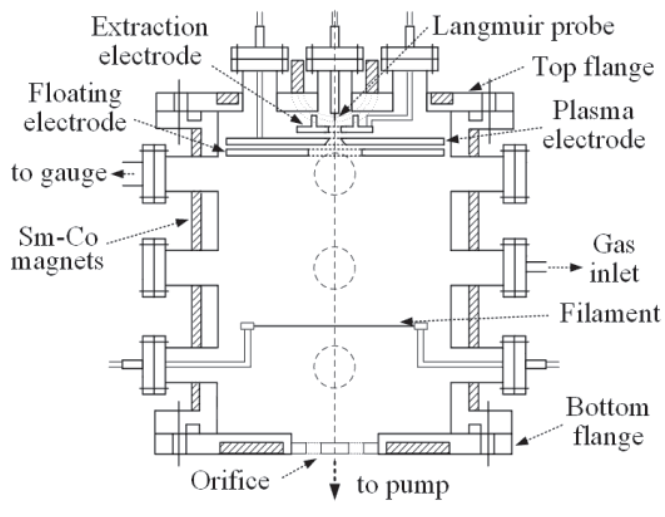

Fig. 1 The schematic illustration of the ion source.

tween $\mathrm{W}$ and Ta filament operations. Plasma parameters were measured with a Langmuir probe as a function of the distance from the extraction region.

\section{Experimental Setup}

Filament arc discharges are generated in a cylindrical ion source. Figure 1 displays a schematic diagram of the ion source chamber. The chamber has a volume of $150 \mathrm{~mm}$ diameter times $200 \mathrm{~mm}$ length. Plasma confinement magnets produced alternating magnetic field along the longitudinal direction on the outside wall forming a multi-cusp magnetic field. A $0.5 \mathrm{~mm}$ diameter and $70 \mathrm{~mm}$ length liner filament is negatively biased at $80 \mathrm{~V}$ against the chamber wall anode in $0.5 \mathrm{~Pa}$ hydrogen filling gas. The filament is fixed at a distance of $75 \mathrm{~mm}$ from the bottom flange. A stainless-steel plasma electrode masked by a $1.5 \mathrm{~mm}$ thick of floating electrode, exposes the surface in the center circular region of $30 \mathrm{~mm}$ diameter to the hydrogen plasma. The floating electrode is separated with $1 \mathrm{~mm}$ distance from the plasma electrode by ceramic washers. Negatively charged particles, i.e. electrons and $\mathrm{H}^{-}$ions are extracted by an extraction electrode with the voltage of $800 \mathrm{~V}$. A Langmuir probe is inserted through holes of $5 \mathrm{~mm}$ diameter of the plasma electrode and the extraction electrode to the plasma. The $0.35 \mathrm{~mm}$ diameter and $4 \mathrm{~mm}$ length tip of the Langmuir probe is made of W. In the experiment of $\mathrm{H}^{-}$ion extraction, negative ion current was detected by a Faraday cup introduced from the upper side of the extraction electrode by replacing the probe.

\section{Experimental Results}

\subsection{Electron temperature}

Probe characteristics with respect to the distance from the extractor were measured by the movable Langmuir probe inserted into the hot filament cathode discharge of 1.0 A. The distance of $0 \mathrm{~mm}$ represents the surface plane of the floating electrode. The bias voltage was changed from 0 to $2.5 \mathrm{~V}$ in every $0.5 \mathrm{~V}$ interval. Figure 2 shows spatial distributions of the electron temperature $\left(T_{e}\right)$ along
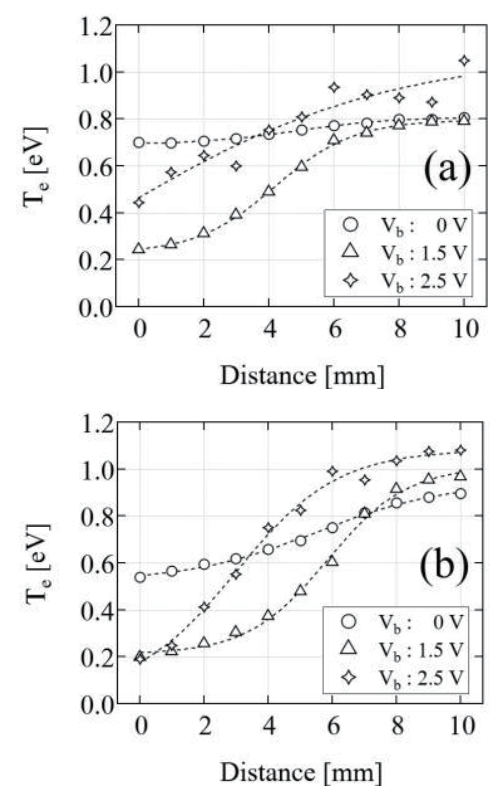

Fig. 2 The spatial distributions of the electron temperature: (a) W filament, (b) Ta filament.

the extraction axis. Lower $T_{e}$ was observed in the vicinity of the extraction region than the region far from the plasma electrode. In comparison with the $\mathrm{W}$ filament, the result of the Ta filament showed steep characteristics of $T_{e}$ with the distance. The operation of the Ta filament also indicated lower $T_{e}$ regardless of the bias voltage at the nearest point for the extractor, while higher $T_{e}$ appeared around $10 \mathrm{~mm}$ distance from the plasma electrode.

\subsection{Electron density}

Electron density $\left(n_{e}\right)$ measured in the extraction region is plotted in Fig. 3. The $n_{e}$ increased as the distance from the plasma electrode increased. The result shows slow linear increase toward the plasma center at $0 \mathrm{~V}$ bias, whereas the regions of steep slope appear as the bias voltage is increased from $0 \mathrm{~V}$ to $2.5 \mathrm{~V}$. The Ta filament operation indicated slightly lower $n_{e}$ with respect to the $\mathrm{W}$ filament. The spatial distributions for the change in bias voltage showed the differences between $\mathrm{W}$ and Ta filament; $n_{e}$ at $0 \mathrm{~mm}$ for $1.5 \mathrm{~V}$ bias voltage was $5 \times 10^{9} \mathrm{~cm}^{-3}$ lower than that of $0 \mathrm{~V}$ for the $\mathrm{W}$ filament operation, while Ta showed the difference of $2 \times 10^{9} \mathrm{~cm}^{-3}$ between the corresponding conditions.

\subsection{Plasma potential}

The plasma potential $\left(V_{p}\right)$ against the distance are shown in Fig. 4. The potential in the vicinity of the extractor increased as the increment of the bias voltage. The spatial profile of the plasma potential changed from increasing to decreasing toward the plasma center (larger distance) as the bias voltage increased. In the region far from the extractor, potential of the plasma generated by $\mathrm{W}$ filament converged with respect to the bias voltage from 0 to $2.5 \mathrm{~V}$. 

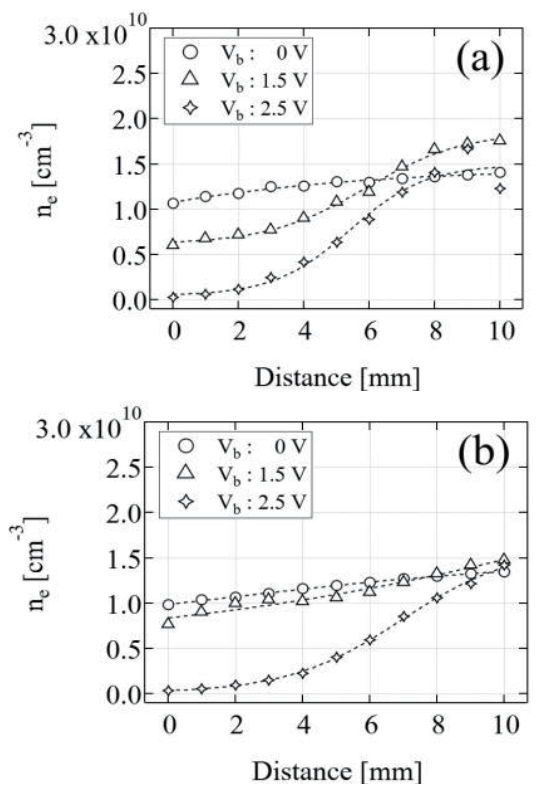

Fig. 3 The spatial distributions of the electron density: (a) W filament, (b) Ta filament.
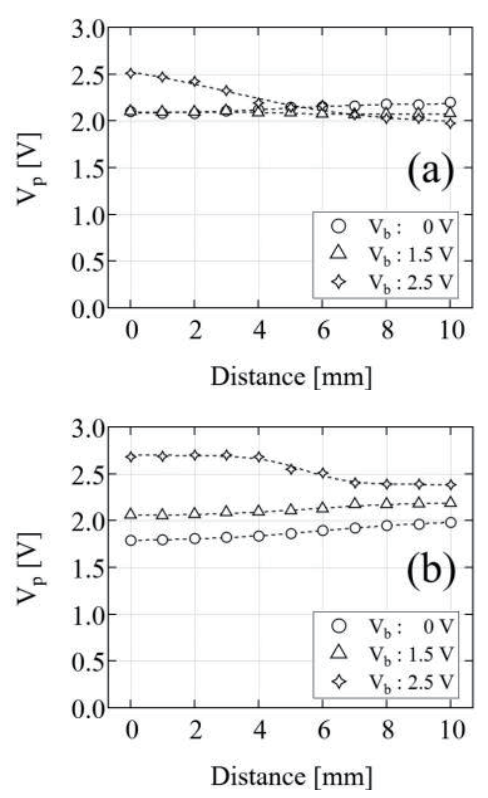

Fig. 4 The spatial distributions of the plasma potential: (a) W filament, (b) Ta filament.

For the Ta filament, however, the potential at $10 \mathrm{~mm}$ increased corresponding to increment of the bias voltage.

\subsection{Electron and ion saturation current}

Figures 5 and 6 exhibit the spatial distributions of electron saturation current $\left(I_{e s}\right)$ and those of the ion saturation current $\left(I_{i s}\right)$, respectively. The ion saturation currents were obtained as the probe current values at $-8 \mathrm{~V}$ probe bias voltage. As the bias voltage increased, the slopes of both curves of saturation currents became steeper. In terms of filament material comparison, Ta showed similar
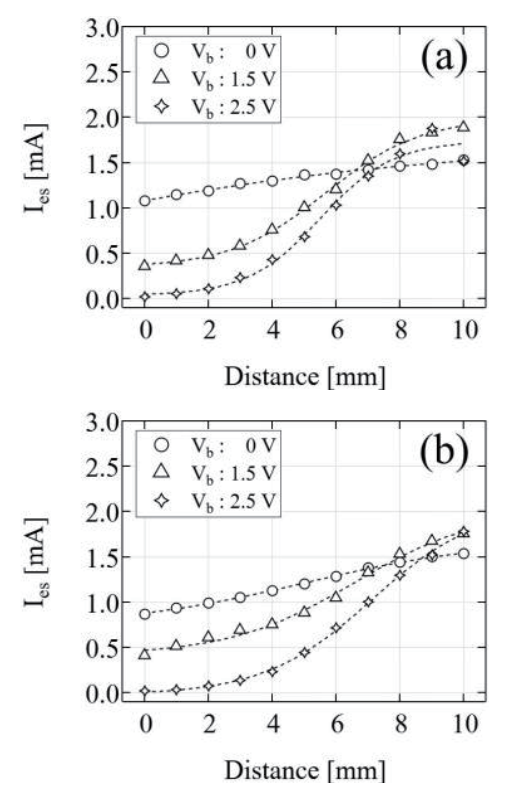

Fig. 5 The spatial distributions of the electron saturation current: (a) W filament, (b) Ta filament.
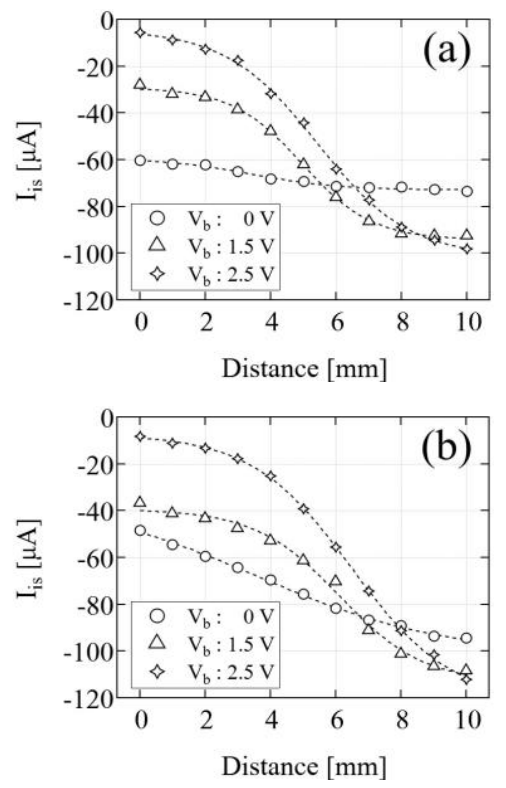

Fig. 6 The spatial distributions of the ion saturation current: (a) W filament, (b) Ta filament.

amount of $I_{e s}$ but higher absolute intensity of $I_{i s}$ with respect to $\mathrm{W}$ in the region far from the plasma electrode.

\section{Discussion}

The probe measurements showed different characteristics in the region close to the plasma electrode between $\mathrm{W}$ and Ta filament operations. According to the result of the Ta filament, the lower electron temperatures were observed for each bias voltage in the vicinity of the plasma electrode, comparing with the $\mathrm{W}$ filament. In the volume process of the $\mathrm{H}^{-}$ion production, low energy electrons can be 
the seeds of dissociative electron attachment reaction to vibrationally excited molecules. Namely, the plasma driven by Ta filament has high potential to $\mathrm{H}^{-}$ion yield from dissociative attachment. The higher density of low temperature electrons in the Ta operation can be attributed to frequent collisions between electrons and hydrogen atoms and/or molecules. The density of the atomic hydrogens near the plasma electrode can be lower than $\mathrm{W}$ filaments due to the enhanced atomic hydrogen adsorption of Ta deposited on the plasma electrode surface by evaporation from Ta filaments. Consequently, the density of hydrogen molecules including vibrationally excited molecules may become high in the extraction region. The old results reported by Hall et al. [12] show the higher vibrational levels are populated for a discharge maintained with Ta filament. These vibrational molecules can form a region of sink for electrons with proper kinetic energy; the electrons are transferred to $\mathrm{H}^{-}$in the region increasing the ratio of $I_{i s} / I_{e s}$. The larger concentration of excited molecules near the plasma electrode can reduce the local electron temperature through collisions.

The difference of electron temperature can affect the co-extracted electron current; the average Larmor radius of electrons, which should directly affect the cross-field electron transport to the extraction aperture becomes larger at higher electron temperature. As shown in [7], the amount of co-extracted electrons makes the $I_{H^{-}} / I_{\text {ext }}$ ratio large at higher plasma electrode bias $V_{b}$. Operation with Ta filament made this ratio higher for entire range of $V_{b}$ compared with $\mathrm{W}$ filament operation. Thus, the extraction characteristics of negatively charged particles can be explained by the measured plasma parameters.

The spatial distribution of the plasma potential changes responding to the applied bias voltage to the plasma electrode. The $\mathrm{H}^{-}$ion transport and electron transport toward the extractor, or the plasma grid are sensitively affected by this electrical potential profile. As the result of [7], both $\mathrm{W}$ and Ta filament operation indicated the maximum negative ion current at $1.8 \mathrm{~V}$ bias voltage. From the result of the plasma potential measurement in Fig. 4, the potential structures at $1.8 \mathrm{~V} V_{b}$ exhibit plateau from the extraction region to the driver region. For a bias voltage higher than $1.8 \mathrm{~V}$, the direction of the electric field not only attracts $\mathrm{H}^{-}$ions but also electrons toward the extraction electrode, reducing electron density near the plasma electrode as shown in Fig. 3. Contrary to the extractor region, both the electron density and the temperature increased at the deeper region of the ion source plasma at the positive $V_{b}$ bias.

No significant difference was observed between W and Ta filament operation in the spatial distribution of electron density. Meanwhile, the difference of the electron density as well as the electron density can affect the $\mathrm{H}^{-}$ion transport in a very weak electrical potential as the function of Debye shielding length. The parameter $\left(T_{e} / n_{e}\right)^{1 / 2}$ was calculated from Figs. 2 and 3 to show it for compar-
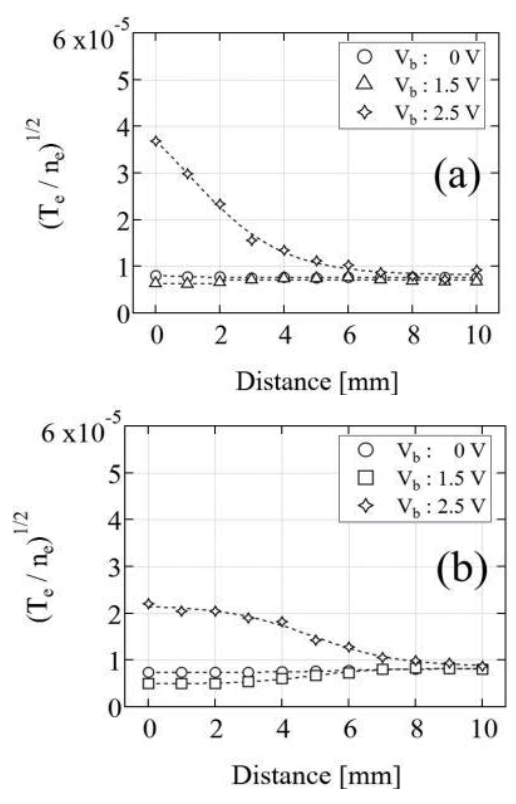

Fig. 7 The function of Debye length calculated from Fig. 3 and Fig. 4: (a) W filament, (b) Ta filament.
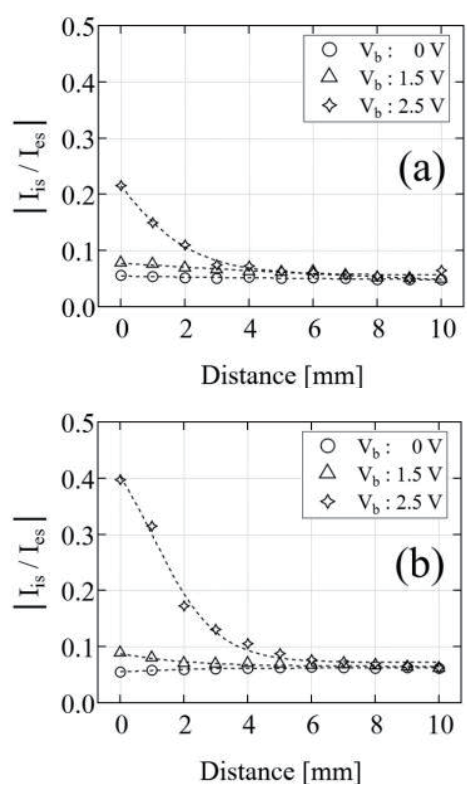

Fig. 8 The absolute ratio of ion saturation current to electron saturation current: (a) W filament, (b) Ta filament.

ison between the Ta filament operation and the $\mathrm{W}$ filament in Fig. 7. The shorter Debye length in the vicinity of the plasma electrode can suppress penetration of extraction electric field into the plasma, and Fig. 7 indicates some smaller penetration of the field for the Ta filament operation.

The present analyses were carried out based upon probe theory for pure electron plasma assuming the electron concentration predominates the negative charged species in the plasma. An extensive care is required for a probe $I-V$ curve analysis when the concentration of neg- 
ative ions in plasma is substantial [13]. Apart from the effect due to a magnetic field [14], the ratio of positive ion saturation current, $I_{i s}$, to negative ion/electron saturation current, which appears as the electron saturation current in the ordinary $I-V$ characteristics, $I_{e s}$, was plotted as a function of distance. Figure 8 summarizes the ratios for both $\mathrm{W}$ filament and Ta filament operations for different bias voltage induced to the plasma electrode. The figures (a) and (b) show positive ion saturation currents stay less than $20 \%$ of $I_{e s}$, and show the existence of enough electrons to estimate the temperature. Figure 8 (b) also indicates that the relative concentration of $\mathrm{H}^{-}$can be substantially higher at the positive $V_{b}$.

\section{Conclusion}

The plasma parameters including the potential structure in the extraction region of the $\mathrm{H}^{-}$ion source were compared between a DC plasma driven by a W filament and that by a Ta filament. The operation with the Ta filament showed spatial distributions of electron temperature, plasma potential and ratio of the saturation current different from the operation with $\mathrm{W}$ filament when these parameters were plotted as functions of the distance. The lower electron temperature, which can affect the amount of $\mathrm{H}^{-}$ions produced by dissociative electron attachment reaction, can be the result of recombinative desorption of incident atomic hydrogen. Adsorption of hydrogen atoms by Ta deposited on the plasma electrode surface can also reduce $\mathrm{H}^{-}$ion destruction near the plasma electrode surface. In addition to the low electron temperature, shorter Debye shield length associated with Ta filament operation can increase the ratio of $\mathrm{H}^{-}$ion current to co-extracted electron current by suppressing electrons extraction. The current results qualitatively explain the observed advantage of the
Ta filament operation against the $\mathrm{W}$ filament operation of the $\mathrm{H}^{-}$ion source. To make the discussion quantitative, a local distribution of vibrational/rotational levels of hydrogen molecule at the vicinity of the plasma electrode [15] is indispensable.

\section{Acknowledgments}

This work has been supported in part by Collaborative Research Program of National Institute for Fusion Science NIFS18KNTR003, and in part by Japan Society for Promotion of Science KAKENHI No. 17H03512.

[1] M. Bacal and M. Wada, Appl. Phys. Rev. 2, 021305 (2015).

[2] L. Schiesko et al., Plasma Source Sci. Technol. 17, 035023 (2008).

[3] Yu.I. Belchenko, G.I. Kuznetsov and E.A. Grigoryev, Rev. Sci. Instrum. 71, 1079 (2000).

[4] M. Sasao et al., Appl. Ohys. Express 11, 066201 (2018).

[5] M. Nishiura et al., Rev. Sci. Instrum. 73, 949 (2002).

[6] M. Bacal and M. Wada, AIP Conf. Proc. 1869, 030025 (2017).

[7] S. Masaki, K. Maeshiro, K. Tsumori and M. Wada, AIP Conf. Proc. 2052, 020002 (2018).

[8] K.N. Leung and M. Bacal, Rev. Sci. Instrum. 55, 338 (1984).

[9] K.N. Leung, K.W. Ehlers and M. Bacal, Rev. Sci. Instrum. 54, 56 (1983).

[10] H. Takahashi, T. Kasuya and M. Wada, Rev. Sci. Instrum. 77, 03A513 (2006).

[11] K. Tsumori et al., Rev. Sci. Instrum. 87, 02B936 (2016).

[12] I. Hall, I. Cadez, M. Landau, F. Pichou and C. Scherman, Phys. Rev. Lett. 60, 337 (1988).

[13] E. Stamate and K. Ohe, J. Appl. Phys. 84, 2450 (1998).

[14] M. Sugawara, Phys. Fluids 9, 797 (1966).

[15] T. Mosback, Plasma Sources Sci. Technol. 14, 610 (2005). 